\title{
Efficient and Spectrally Stable Blue Perovskite Light-Emitting Diodes Based on Potassium Passivated Nanocrystals
}

Fei Yang, Hongting Chen, Rui Zhang, Xiaoke Liu, Weizhuo Zhang, JiBin Zhang, Feng Gao and Lei Wang

The self-archived postprint version of this journal article is available at Linköping University Institutional Repository (DiVA):

http://urn.kb.se/resolve?urn=urn:nbn:se:liu:diva-163634

N.B.: When citing this work, cite the original publication.

Yang, F., Chen, H., Zhang, R., Liu, X., Zhang, W., Zhang, J., Gao, F., Wang, L., (2020), Efficient and Spectrally Stable Blue Perovskite Light-Emitting Diodes Based on Potassium Passivated Nanocrystals, Advanced Functional Materials, , 1908760. https://doi.org/10.1002/adfm.201908760

Original publication available at:

$\underline{\text { https://doi.org/10.1002/adfm.201908760 }}$

Copyright: Wiley (12 months)

http://eu.wiley.com/WileyCDA/ 


\section{WILEY-VCH}

DOI: 10.1002/ ((please add manuscript number))

\section{Article type: Full Paper}

\section{Efficient and Spectrally Stable Blue Perovskite Light-emitting Diodes based on Potassium Passivated Nanocrystals}

Fei Yang ${ }^{l}$, Hongting Chen ${ }^{1,2}$, Rui Zhang ${ }^{1}$, Xiaoke Liu ${ }^{2}$, Weizhuo Zhang ${ }^{1}$, JiBin Zhang ${ }^{1}$, Feng $\mathrm{GaO}^{2 *}$ and Lei Wang ${ }^{1 *}$

1 Wuhan National Laboratory for Optoelectronics, Huazhong University of Science and Technology, Wuhan 430074, China

2 Department of Physics, Chemistry and Biology (IFM), Linköping University, Linköping 58183, Sweden

E-mail: wanglei@mail.hust.edu.cn;

E-mail: feng.gao@liu.se

Keywords: perovskite light-emitting diodes, defects passivation, surface ligands, charge injection, spectrally stable

Metal halide perovskites have aroused tremendous interest in the past several years for their promising applications in display and lighting. However, the development of blue perovskite light-emitting diodes (PeLEDs) still lags far behind that of their green and red cousins due to the difficulty in obtaining high-quality blue perovskite emissive layers. In this study, we conceive a simple approach to improve the emission and electrical properties of blue perovskites. By introducing an alkali metal ion to occupy some sites of peripheral suspended organic ligands, the non-radiative recombination is suppressed, and consequently, blue $\mathrm{CsPb}(\mathrm{Br} / \mathrm{Cl})_{3}$ nanocrystals with a high photoluminescence quantum efficiency of $38.4 \%$ are obtained. The introduced $\mathrm{K}^{+}$acts as a new type of metal ligand, which not only suppresses nonradiative pathways but also improves the charge carrier transport of the perovskite nanocrystals. With further engineering of the device structure to balance the charge injection rate, a spectrally stable and efficient blue PeLED with a maximum external quantum efficiency of $1.96 \%$ at the emission peak of $477 \mathrm{~nm}$ is fabricated. 


\section{WILEY-VCH}

\section{Introduction}

Metal halide perovskites have been widely used in solar cells, lasers, detectors and lightemitting diodes (LEDs), owing to their readily tunable optical bandgaps, narrow full width at half-maximum (FWHM), low cost, solution processability and superior optical and electrical properties. ${ }^{[1-8]}$ Recently, encouraging progress has been made in near-infrared, red and green perovskite LEDs (PeLEDs), in which the external quantum efficiencies (EQEs) have been boosted to more than $20 \%{ }^{[9-11]}$ Efficient LEDs for all the three basic colors are required to realize their practical application in display and light sources. However, blue PeLEDs still lag behind the red and green ones due to the difficulty in fabricating an efficient and stable perovskite emissive layer and the lack of proper charge transport material to achieve the desirable device structure to reduce the charge injection barrier. ${ }^{[12]}$ The current strategies to develop blue perovskite films can be categorized into three methods. The first method is the use of mixed-halide perovskites. ${ }^{[13-16]}$ Because of the halide segregation, ${ }^{[17,18]}$ an irreversible emission shift can occur during the bias operation in these mixed-halide-based blue PeLEDs. The second method is the use of perovskite nanoplatelets by inserting large organic cations to form a quasi-two-dimensional (quasi-2D) phase. ${ }^{[19]}$ For example, by engineering the perovskite composition, Jin and coworkers developed energetically coupled quantum-confined nanoparticles and quasi-2D phases to achieve a record EQE of 9.5\% for blue PeLEDs. ${ }^{[20]}$ However, as multiple phases can coexist in the perovskite films, these quasi-2D perovskites show broad emission peaks. ${ }^{[21]}$ The last method is the use of tiny perovskite nanocrystals (NCs) with a strong quantum effect, whose emission can be easily tuned to deep blue. ${ }^{[22]}$ However, blue perovskite NCs always exhibit low photoluminescence quantum yield (PLQY) because of enormous traps. ${ }^{[23]}$ In addition, a long-chain organic ligand is essential to synthesize perovskite NCs. However, these insulating organic components will hinder the charge transport within the device, which hampers the performance of the LEDs. ${ }^{[24]}$ Moreover, the commonly used long chain organic ligands are amine and acid, which can result in the desorption of surface ligands 


\section{WILEY-VCH}

and defects due to the protonation process between acid and amine. ${ }^{[25,26]}$ Sargent and his coworkers proposed a new method to synthesize amine-free perovskite quantum dots to avoid this phenomenon successfully. ${ }^{[27]}$ Moreover, introducing inorganic ligands to replace a part of organic ligands, which can enhance the charge transport and reduce the non-radiative recombination, can be an effective method to overcome the aforementioned challenges. ${ }^{[24,28,29]}$ In this paper, we demonstrate the use of potassium to obtain efficient and stable blue $\mathrm{CsPb}(\mathrm{Br} / \mathrm{Cl})_{3} \mathrm{NCs}$. With the introduction of a rational amount of $\mathrm{K}^{+}$, which can strongly bond with the halogen anion to form surface passivation to reduce the non-radiative recombination, the resulting perovskite NCs show not only improved PLQY but also stability. In addition, as the ionic $\mathrm{KBr}$ (or $\mathrm{KCl}$ ) can be regarded as surface ligands capping the perovskite NCs, the longchain organic ligands are dramatically reduced, which is beneficial to the charge transport of the perovskite films. With further optimization of the device structure to balance the charge injection, a spectrally stable and efficient blue PeLED with an EQE of 1.96 is achieved, which shows evident improvement compared with a control device. Our method is a new route to fabricate high-quality blue perovskite NCs, paving the way for further improvement of the performance of blue PeLEDs.

\section{Results and Discussion}

\subsection{Structure Characterization}

The perovskite NCs are synthesized using a well-established ligand-assisted-reprecipitation method. (See schematic illustrated in Figure S1, Supporting Information). For $\mathrm{K}^{+}$-based NCs, different ratios of $\mathrm{K}_{2} \mathrm{CO}_{3}$ in octanoic acid (OTAc) are added into a cesium precursor solution (See Experimental Section for details). Here, we define $x=[\mathrm{K}] /[\mathrm{Pb}]$, where $x$ represents the molar ratio of potassium to lead source in the $\mathrm{K}^{+}$-incorporated samples. First, we use inductively- coupled plasma optical emission spectrometry to determine the real chemical compositions of the perovskite NCs with the introduction of different ratios of potassium. The original added potassium concentrations $([\mathrm{K}] /[\mathrm{Pb}])$ are $2.4 \%, 4.0 \%$, and $8.0 \%$, and the finally 


\section{WILEY-VCH}

determined $[\mathrm{K}] /[\mathrm{Pb}]$ concentrations in potassium-based NC samples are $2.1 \%, 2.6 \%$, and $4.3 \%$, respectively, which reveal that $\mathrm{K}^{+}$only partially exists in the final perovskite NCs (Table S1). Previous reports have shown that an alkali metal ion can be incorporated into the perovskite lattice. ${ }^{[30-32]}$ X-ray diffraction (XRD) and transmission electron microscopy (TEM) measurements are performed to investigate the effects of $\mathrm{K}^{+}$on the structural properties of the resulting NCs. All the samples show evident diffraction peaks around $2 \theta=15.00^{\circ}, 21.00^{\circ}$, $31.00^{\circ}, 34.00^{\circ}, 38.00^{\circ}$, and $44.00^{\circ}$, corresponding to the (100), (110), (200), (210), (211) and (220) crystal planes of the cubic $\mathrm{CsPb}(\mathrm{Br} / \mathrm{Cl})_{3}$ phase, respectively (Figure 1a). No diffraction peak shift can be observed in the $\mathrm{K}^{+}$-based samples, suggesting that $\mathrm{K}^{+}$remains outside the perovskite NCs rather than being incorporated into the perovskite lattice. Otherwise, diffraction peak shift toward a higher angle would occur as the smaller $\mathrm{K}^{+}$ions can cause lattice contraction. ${ }^{[33]}$ This phenomenon is consistent with the recent studies on potassium doping for obtaining efficient perovskite solar cells. ${ }^{[34,35]}$ However, we observe a broad FWHM at the diffraction peak of the (200) plane after the incorporation of $\mathrm{K}^{+}$. According to the Scherrer equation, ${ }^{[36]}$ an increased FWHM may indicate decreased size of the perovskite NCs. The TEM images in Figure 1 indicate monodispersed and nearly cubic perovskite NCs with the introduction of $\mathrm{K}^{+}$. The inserted high-resolution TEM (HR-TEM) images in Figure $\mathbf{1 b}$ and $\mathrm{c}$ show the lattice plane of (200) and (211), respectively, for pristine and $\mathrm{K}^{+}$-based $\mathrm{CsPb}(\mathrm{Br} / \mathrm{Cl})_{3}$ NCs, which further indicates that the addition of $\mathrm{K}^{+}$does not influence the crystal structure of NCs. The NCs exhibit a slight increase in size with the incorporation of $\mathrm{K}^{+}$, as shown in the size distribution histograms in Figure 1d and e, where the average sizes of the pristine and $\mathrm{K}^{+}$based $\mathrm{CsPb}(\mathrm{Br} / \mathrm{Cl})_{3} \mathrm{NCs}$ are $9.94 \mathrm{~nm}$ and $10.92 \mathrm{~nm}$, respectively. This result appears inconsistent with our XRD measurement; however, it can be observed that the FWHM of the size distributions also increases with the incorporation of $\mathrm{K}^{+}$. In other words, in pristine $\mathrm{NCs}$, some large NCs can also exist, which may result in slightly broadened FWHM at the XRD peak. Previous reports have demonstrated that metal ion doping strategies can accelerate the speed of 


\section{WILEY-VCH}

the reaction, leading to an increase in the size of the NCs. ${ }^{[30]}$ In this case, we hypothesis that, due to the desorption of surface organic ligands, ${ }^{[37]}$ the introduced inorganic salt can influence the size of the NCs, which would be discussed later.

\subsection{Effects of Potassium Ions}

To investigate further how the introduction of $\mathrm{K}^{+}$influences the size of the NCs, we hypothesize that the introduced $\mathrm{K}^{+}$can bond with the halide ions in the $\mathrm{NC}$ solution to form an inorganic ligand capped outside the surface of the NCs. Consequently, the organic ligands are reduced, leading to an increased size of the NCs. ${ }^{[37]}$ Accordingly, Fourier-transform infrared spectroscopy (FTIR) measurement is performed to investigate the evolution of organic ligands after introducing $\mathrm{K}^{+}$. As shown in Figure S2, the strong peaks at 2700-3000 $\mathrm{cm}^{-1}, 1466 \mathrm{~cm}^{-1}$, and $1720 \mathrm{~cm}^{-1}$ can be attributed to the $\mathrm{C}-\mathrm{H}$ bending vibration and $\mathrm{C}=\mathrm{O}$ stretching of didodecyldimethylammonium bromide $(\mathrm{DDAB})$ and OTAc, respectively. ${ }^{[38,39]}$ With the introduction of $\mathrm{K}^{+}$, the typical peak intensity of organic ligands is reduced, indicating the reduction of organic ligands in the perovskites. Furthermore, we conduct X-ray photoelectron spectroscopy (XPS) measurements to explore the effect of $\mathrm{K}^{+}$on the electronic states of the main elements, as shown in Figure S3. The XPS spectra of both the pristine and $\mathrm{K}^{+}$-based NCs show the presence of $\mathrm{Cs}, \mathrm{Pb}, \mathrm{Br}, \mathrm{Cl}, \mathrm{N}, \mathrm{C}$, and $\mathrm{O}$ elements (Figure S3a). It can be observed that the peaks of $\mathrm{Br} 3 \mathrm{~d}, \mathrm{Cl} 2 \mathrm{p}$, and $\mathrm{Pb} 4 \mathrm{f}$ in the $\mathrm{K}^{+}$-based NCs shift to lower energy compared with those for the pristine one, indicating a different chemical bonding environment of $\mathrm{Br}, \mathrm{Cl}$, and $\mathrm{Pb}$ elements, whereas the peak of $\mathrm{Cs} 3 \mathrm{~d}$ remains invariant (Figure 2a, $2 \mathrm{~b}$ and Figure S3).

Based on the XRD results and the peak shift in XPS, we speculate that $\mathrm{K}^{+}$is mainly anchored on the surface of NCs through bonding with halogen anion. In addition, we extract the $\mathrm{O} / \mathrm{Pb}$ and $\mathrm{N} / \mathrm{Pb}$ and from the XPS data (Figure $2 \mathrm{c}$ and $2 \mathrm{~d}$ ), which show that the atomic ratio of $\mathrm{O} / \mathrm{Pb}$ drastically declined from 1.64 to 0.4 with the incorporation of $\mathrm{K}^{+}$. As the $\mathrm{O}$ element only exists in OTAc, the amount of OTAc severely decreases. According to previous reports, the tetraoctylammonium bromide added here serves as a solubilizing agent and halide source rather 


\section{WILEY-VCH}

than a surface ligand due to its large steric hindrance ${ }^{[28,40]}$. Therefore, the decrease in the N/Pb ratio indicates the decreasing amount of DDAB. From the XPS data, the atomic ratio of $\mathrm{Cs} /\left(\mathrm{Br}+\mathrm{Cl}\right.$ ) could be calculated (Table S2). For both pristine and $\mathrm{K}^{+}$-based NCs, the atomic ratio of $\mathrm{Cs} /(\mathrm{Br}+\mathrm{Cl})$ remains invariant $(0.142)$, which is yet another evidence proving that $\mathrm{K}^{+}$ remains outside the perovskite NCs rather than substitute the $\mathrm{Cs}^{+}$site.

The XPS results are consistent with the FTIR results, demonstrating that the addition of $\mathrm{K}^{+}$ causes a decrease in the organic ligands on the surface of the perovskite NCs. Based on the above discussion, we can claim that $\mathrm{K}^{+}$can bond with halide ions to form metal ligands anchored on the surface of NCs, leading to a decrease in the amount of insulating organic ligands, as schematically illustrated in Figure 3e. Consequently, the deposited perovskite films also show a different film morphology, as shown by the atomic force microscopy (AFM) images in Figure S4. Due to a large number of organic ligands, the pristine perovskite film shows a large root-mean-square (RMS) roughness of $8.69 \mathrm{~nm}$. In contrast, owing to the reduced number of organic ligands, the $4.0 \% \mathrm{~K}^{+}$-based film shows a decreased RMS value of $6.21 \mathrm{~nm}$, which guarantees a low leak current density in an LED device. Upon further increasing the $\mathrm{K}^{+}$molar ratio to $8.0 \%$, there is a serious aggregation (as depicted by the red circle in Figure S4d) with a large RMS of $10.50 \mathrm{~nm}$. It can be concluded that the introduction of a rational amount of $\mathrm{K}^{+}$ improves the surface morphology of $\mathrm{CsPb}(\mathrm{Br} / \mathrm{Cl})_{3} \mathrm{NC}$ films, whereas excessive $\mathrm{K}^{+}$will degrade the surface morphology, resulting in a bumpy surface. The variation in the surface morphology of films with different values of $x$ can be attributed to the growth speed of NCs, where more $\mathrm{K}^{+}$ leads to faster growth. ${ }^{[41]}$ Therefore, the amount of $\mathrm{K}^{+}$should be strictly controlled to obtain the desirable surface morphology.

\subsection{Defects Passivation of Potassium}

Figure 3a shows the ultraviolet-visible (UV-vis) absorption and photoluminescence (PL) spectra of perovskite NC solutions with different $x$ values, in which a gradually red-shift can be observed with the increase in the $\mathrm{K}^{+}$ratios. Consequently, the corresponding optical band gap 


\section{WILEY-VCH}

shows a slight decrease as displayed in Figure S5. This may be ascribed to the formation of slightly larger NCs caused by the decrease in the amount of organic ligands as discussed before. ${ }^{[42]}$ Insets in Figure 3a are the corresponding photographs of NC dispersions under daylight and $365 \mathrm{~nm}$ UV lamp. For perovskite NCs, surface ligands are essential to stabilize the NCs in the solution for preventing self-aggregation and to passivate the surface defects. ${ }^{[6]}$ However, the current ligands used to synthesis high-quality perovskite NCs are amine and acid, which can result in the desorption of surface ligands and defects due to the protonation process between acid and amine. ${ }^{[25,26]}$ Fortunately, owing to the much stronger ionic bond, the $\mathrm{K}^{+}$-based perovskite NCs show considerably improved stability and reduced non-radiative recombination. As shown in Figure S6, the $\mathrm{K}^{+}$-based NC solution can retain its initial PL properties without any precipitate at the bottom after storage for $24 \mathrm{~h}$, and strong blue emission can be observed under the irradiation of a $365 \mathrm{~nm}$ UV lamp. However, for pristine NCs, the colloidal solution becomes cloudy after storage for $24 \mathrm{~h}$, which might be related to the loss of highly dynamic organic ligands. ${ }^{[25]}$ Consequently, it exhibits a significantly weaker blue emission compared with that of the $\mathrm{K}^{+}$-based samples.

We firstly measure the PLQY of the NC solutions to investigate the non-radiative recombination in the NCs further (Figure 2b). By increasing the $\mathrm{K}^{+}$molar ratio, the PLQY of the NC solutions is enhanced from $9.50 \%(x=0)$ to $38.4 \%(x=8.0 \%)$. The time-resolved PL decay measurement also confirms the much- reduced non-radiative recombination with the incorporation of $\mathrm{K}^{+}$, as shown in Figure 2c. We fit the decay curves by a bi-exponential function and the corresponding data are listed in Table S3. The fitted PL lifetime of the $\mathrm{K}^{+}$-based NCs is much- longer than that of pristine NCs, which indicates stronger radiative recombination.

\subsection{Device Performance}

LEDs with a structure of indium tin oxide (ITO)/poly(ethylenedioxythiophene):polystyrene sulfonate (PEDOT:PSS)/poly(N,N'-bis(4-butylphenyl)-N,N'-bisphenylbenzidine) (poly$\mathrm{TPD}) / \mathrm{CsPb}(\mathrm{Br} / \mathrm{Cl})_{3} \mathrm{NCs} /(1,3,5$-Tris (1-phenyl-1H-benzimidazol-2-yl)benzene) (TPBi)/LiF/Al 


\section{WILEY-VCH}

are fabricated to investigate the effect of different concentrations of $\mathrm{K}^{+}$on the performance of the PeLEDs. The energy level diagram of the device and the related electroluminescence (EL) characterization is presented in Figure S7. The valance band of the perovskite films is confirmed via ultraviolet photoelectron spectroscopy (UPS) as shown in Figure S8, whereas the others are obtained from literatures. ${ }^{[15,43]}$ The current density-voltage-luminance curves are shown in Figure S7b, where a higher current density and luminescence can be observed in the $\mathrm{K}^{+}$-based devices. The much- higher current density in the $\mathrm{K}^{+}$-based LEDs also indicates the superior conductivity of the perovskite films due to the reduced insulating organic ligands. It is further verified through the hole-only devices (ITO/PEDOT:PSS/poly-TPD/NCs/ $\mathrm{MoO}_{3} / \mathrm{Al}$ ) in Figure S9, where enhanced current can be observed with the increase in the $\mathrm{K}^{+}$ratios. ${ }^{[44]}$ The champion device with $x=4.0 \%$ yields the highest EQE of $1.19 \%$, as shown by the electrical output parameters summarized in Table 1. Moreover, the champion device shows a typical $\mathrm{T}_{50}$ lifetime of $4.5 \mathrm{~min}$, which is 2.62 times longer than that of the LED device based on the pristine NCs (Figure S10). As previously mentioned, further increasing the addition ratio $x$ to $8.0 \%$ can yield higher PLQY; however, the poor surface morphology results in a slightly reduced performance. In addition, the EL spectra demonstrate a red-shift with the increase in the $\mathrm{K}^{+}$ amount (Figure S7d), which is consistent with the UV-vis spectra and PL behavior as discussed before. However, the EL emission remains in the blue region, as illustrated by the CIE (Commission Internationale de l'Eclairage) coordinates in the insert in Figure S7d.

For an LED device, any unbalanced charge injection can cause charge accumulation at the perovskite interface, degrading the device performance. In this case, due to the deep valence band of the perovskite layer, ${ }^{[45]}$ engineering the device structure with wide-bandgap hole transport layers (HTLs) to facilitate the hole injection is essential to improve the efficiency.

In the above device structure of Figure S7a, poly-TPD and TPBi served as the HTL and electron transport layer (ETL), respectively. However, due to the considerable difference in the charge mobility, where the hole mobility of poly-TPD is $1 \times 10^{-4} \mathrm{~cm}^{2} \mathrm{v}^{-1} \mathrm{~s}^{-1}$ and the electron mobility of 


\section{WILEY-VCH}

TPBi is $1 \times 10^{-6} \mathrm{~cm}^{2} \mathrm{v}^{-1} \mathrm{~s}^{-1},{ }^{[46,47]}$ unbalanced carrier injection could occur in the LED devices. To balance the charge injection, we first replace the TPBi with 2,4,6-Tris(3(diphenylphosphoryl)phenyl)-1,3,5-triazine (PO-T2T), which possesses higher electron mobility $\left(1.1 \times 10^{-4} \mathrm{~cm}^{2} \mathrm{v}^{-1} \mathrm{~s}^{-1}\right)$ and deeper valence band $(-7.5 \mathrm{eV}) \cdot{ }^{[48]}$ Consequently, the maximum EQE is enhanced from $1.19 \%$ to $1.63 \%$ for the champion device (Figure S11). Additionally, we use a bilayer HTL of poly-TPD and poly(9-vinylcarbazole) (PVK) to promote hole injection further. ${ }^{[43]}$ The energy level and schematic diagram of the device are presented in Figure $4 \mathrm{a}$ and $4 \mathrm{~b}$, respectively. Figure $4 \mathrm{c}$ and $4 \mathrm{~d}$ present the current density-voltageluminance and EQE-luminance curves, respectively. The device shows a maximum EQE of $1.96 \%$ and a maximum luminance of $86.95 \mathrm{~cd} / \mathrm{m}^{2}$. To certify the reproducibility, 30 devices of 4.0\% $\mathrm{K}^{+}$-based co-HTLs LED devices have been measured which exhibits an average maximum EQE of 1.89\% (Figure S12). Figure 4e presents the EL spectra of the device driven at the voltage of $3.2 \mathrm{~V}$ and the inset shows the corresponding CIE coordinates. In addition, the co-HTLs-structure-based device shows acceptable spectral stability when the applied voltage increases from 3.2 V to $6.2 \mathrm{~V}$ (Figure 4f). Notably, our device exhibits low efficiency roll-off, indicating a balanced charge injection and recombination. ${ }^{[49]}$ It is worth emphasizing that the stability of the EL spectra is mainly attributed to the balanced charge injection resulting from the co-HTLs structure, ${ }^{[17,50]}$ as $\mathrm{K}^{+}$-based single HTL device undergoes phase segregation under constant voltage (Figure S13). Furthermore, through optimizing the device structure, the optimized blue perovskite NC-LED achieves a state-of-the-art EQE among the blue PeLEDs based on NCs. ${ }^{[12]}$ The performance of the LEDs with $x=4.0 \%$ with different device structures is summarized in Table 2, where devices A, B, and C represent a single HTL device with TPBi as the ETL, a single HTL device with PO-T2T as the ETL, and a stepwise co-HTLs device with PO-T2T as the ETL, respectively.

\section{Conclusion}




\section{WILEY-VCH}

In summary, we demonstrate a spectrally stable and efficient blue PeLED via potassium passivation and device structure engineering. With the incorporation of $\mathrm{K}^{+}$, we reduce not only the non-radiative recombination but also the amount of organic ligand in the resulting perovskite NCs. Owing to the reduced organic compound, the morphology of the deposited perovskite films is improved, leading to enhanced charge transport of the perovskite films. By replacing the TPBi with PO-T2T and applying bi-layer HTLs to engineer the LED architecture further to balance the charge injection, we fabricate a blue PeLED with a champion EQE of $1.96 \%$. This work provides an effective method to improve the quality of perovskite NCs, paving the way toward developing stable and high-efficiency blue PeLEDs in the future.

\section{Supporting Information}

Supporting Information is available from the Wiley Online Library or from the author.

\section{Acknowledgements}

The work was financially supported by the NSFC/China ( 51727809), Wuhan Science and Technology Bureau (2019010701011406). China Postdoctoral Science Foundation (2017M620321). In addition, we wish to thank the support facilities at the Center for Nanoscale Characterization \& Devices, Wuhan National Laboratory for Optoelectronics of Huazhong University of Science and Technology (HUST), the Analytical and Testing Center of HUST and Ceshigo Research Service Agency (www.ceshigo.com).

\section{References}

[1] N. J. Jeon, H. Na, E. H. Jung, T.-Y. Yang, Y. G. Lee, G. Kim, H.-W. Shin, S. Il Seok, J. Lee, J. Seo, Nat. Energy 2018, 3, 682.

[2] C. C. Stoumpos, M. G. Kanatzidis, Adv. Mater. 2016, 28, 5778.

[3] K. Hirose, R. Sinmyo, J. Hernlund, Science 2017, 358, 734. 


\section{WILEY-VCH}

[4] W. Hu, W. Huang, S. Yang, X. Wang, Z. Jiang, X. Zhu, H. Zhou, H. Liu, Q. Zhang, X. Zhuang, J. Yang, D. H. Kim, A. Pan, Adv. Mater. 2017, 29, 1703256.

[5] G. Xing, N. Mathews, S. S. Lim, N. Yantara, X. Liu, D. Sabba, M. Gratzel, S. Mhaisalkar, T. C. Sum, Nat. Mater. 2014, 13, 476.

[6] F. Zhang, H. Zhong, C. Chen, X. G. Wu, X. Hu, H. Huang, J. Han, B. Zou, Y. Dong, ACS Nano 2015, 9, 4533.

[7] X. Li, Y. Wu, S. Zhang, B. Cai, Y. Gu, J. Song, H. Zeng, Adv. Funct. Mater. 2016, 26, 2435

[8] J. Zhang, L. Zhang, P. Cai, X. Xue, M. Wang, J. Zhang, G. Tu, Nano Energy 2019, 62, 434.

[9] K. Lin, J. Xing, L. N. Quan, F. P. G. de Arquer, X. Gong, J. Lu, L. Xie, W. Zhao, D. Zhang, C. Yan, W. Li, X. Liu, Y. Lu, J. Kirman, E. H. Sargent, Q. Xiong, Z. Wei, Nature 2018, 562, 245.

[10] Y. Cao, N. Wang, H. Tian, J. Guo, Y. Wei, H. Chen, Y. Miao, W. Zou, K. Pan, Y. He, H. Cao, Y. Ke, M. Xu, Y. Wang, M. Yang, K. Du, Z. Fu, D. Kong, D. Dai, Y. Jin, G. Li, H. Li, Q. Peng, J. Wang, W. Huang, Nature 2018, 562, 249.

[11] T. Chiba, Y. Hayashi, H. Ebe, K. Hoshi, J. Sato, S. Sato, Y.-J. Pu, S. Ohisa, J. Kido, Nat. Photonics 2018, 12, 681.

[12] N. K. Kumawat, X. K. Liu, D. Kabra, F. Gao, Nanoscale 2019, 11, 2109.

[13] G. Li, F. W. R. Rivarola, N. J. L. K. Davis, S. Bai, T. C. Jellicoe, F. de la Peña, S. Hou, C. Ducati, F. Gao, R. H. Friend, N. C. Greenham, Z.-K. Tan, Adv. Mater. 2016, 28, 3528.

[14] M. K. Gangishetty, S. Hou, Q. Quan, D. N. Congreve, Adv. Mater. 2018, 30, 1706226.

[15] S. Hou, M. K. Gangishetty, Q. Quan, D. N. Congreve, Joule 2018, 2, 2421.

[16] J. Pan, L. N. Quan, Y. Zhao, W. Peng, B. Murali, S. P. Sarmah, M. Yuan, L. Sinatra, N. M. Alyami, J. Liu, E. Yassitepe, Z. Yang, O. Voznyy, R. Comin, M. N. Hedhili, O. F. 


\section{WILEY-VCH}

Mohammed, Z. H. Lu, D. H. Kim, E. H. Sargent, O. M. Bakr, Adv. Mater. 2016, 28, 8718.

[17] I. L. Braly, R. J. Stoddard, A. Rajagopal, A. R. Uhl, J. K. Katahara, A. K. Y. Jen, H. W. Hillhouse, ACS Energy Lett. 2017, 2, 1841.

[18] Y. Zou, Z. Yuan, S. Bai, F. Gao, B. Sun, Materials Today Nano 2019, 5, 100028.

[19] Z. Li, Z. Chen, Y. Yang, Q. Xue, H. L. Yip, Y. Cao, Nat. Commun. 2019, 10, 1027.

[20] Y. Liu, J. Cui, K. Du, H. Tian, Z. He, Q. Zhou, Z. Yang, Y. Deng, D. Chen, X. Zuo, Y. Ren, L. Wang, H. Zhu, B. Zhao, D. Di, J. Wang, R. H. Friend, Y. Jin, Nat. Photonics 2019, 13, 760 .

[21] J. Xing, Y. Zhao, M. Askerka, L. N. Quan, X. Gong, W. Zhao, J. Zhao, H. Tan, G. Long, L. Gao, Z. Yang, O. Voznyy, J. Tang, Z.-H. Lu, Q. Xiong, E. H. Sargent, Nat. Commun. 2018, $9,3541$.

[22] J. Song, J. Li, X. Li, L. Xu, Y. Dong, H. Zeng, Adv. Mater. 2015, 27, 7162.

[23] D. Parobek, B. J. Roman, Y. Dong, H. Jin, E. Lee, M. Sheldon, D. H. Son, Nano Lett. 2016, 16, 7376 .

[24] B.-B. Zhang, S. Yuan, J.-P. Ma, Y. Zhou, J. Hou, X. Chen, W. Zheng, H. Shen, X.-C. Wang, B. Sun, O. M. Bakr, L.-S. Liao, H.-T. Sun, J. Am. Chem. Soc. 2019, 141, 15423.

[25] D. Yang, X. Li, W. Zhou, S. Zhang, C. Meng, Y. Wu, Y. Wang, H. Zeng, Adv. Mater. 2019, 0, 1900767

[26] J. Pan, L. N. Quan, Y. Zhao, W. Peng, B. Murali, S. P. Sarmah, M. Yuan, L. Sinatra, N. M. Alyami, J. Liu, E. Yassitepe, Z. Yang, O. Voznyy, R. Comin, M. N. Hedhili, O. F. Mohammed, Z. H. Lu, D. H. Kim, E. H. Sargent, O. M. Bakr, Adv. Mater. 2016, 28, 8718.

[27] E. Yassitepe, Z. Yang, O. Voznyy, Y. Kim, G. Walters, J. A. Castañeda, P. Kanjanaboos, M. Yuan, X. Gong, F. Fan, J. Pan, S. Hoogland, R. Comin, O. M. Bakr, L. A. Padilha, A. F. Nogueira, E. H. Sargent, Adv. Funct. Mater. 2016, 26, 8757. 


\section{WILEY-VCH}

[28] J. Song, T. Fang, J. Li, L. Xu, F. Zhang, B. Han, Q. Shan, H. Zeng, Adv. Mater. 2018, $30,1805409$.

[29] C. Luo, W. Li, D. Xiong, J. Fu, W. Yang, Nanoscale 2019, 11, 15206.

[30] Y. Liu, G. Pan, R. Wang, H. Shao, H. Wang, W. Xu, H. Cui, H. Song, Nanoscale 2018, $10,14067$.

[31] S. Li, Z. Shi, F. Zhang, L. Wang, Z. Ma, D. Yang, Z. Yao, D. Wu, T.-T. Xu, Y. Tian, Y. Zhang, C. Shan, X. J. Li, Chem. Mater. 2019, 31, 3917.

[32] B. Wang, C. Zhang, S. Huang, Z. Li, L. Kong, L. Jin, J. Wang, K. Wu, L. Li, ACS Applied Materials \& Interfaces 2018, 10, 23303.

[33] H. Liu, Z. Wu, J. Shao, D. Yao, H. Gao, Y. Liu, W. Yu, H. Zhang, B. Yang, ACS Nano 2017, 11, 2239.

[34] M. Abdi-Jalebi, Z. Andaji-Garmaroudi, S. Cacovich, C. Stavrakas, B. Philippe, J. M. Richter, M. Alsari, E. P. Booker, E. M. Hutter, A. J. Pearson, S. Lilliu, T. J. Savenije, H. Rensmo, G. Divitini, C. Ducati, R. H. Friend, S. D. Stranks, Nature 2018, 555, 497.

[35] W. Zhao, Z. Yao, F. Yu, D. Yang, S. Liu, Adv. Sci. 2018, 5, 1700131.

[36] U. Holzwarth, N. Gibson, Nat. Nanotechnol. 2011, 6, 534.

[37] J. H. Park, A. Y. Lee, J. C. Yu, Y. S. Nam, Y. Choi, J. Park, M. H. Song, ACS Appl. Mater. Interfaces 2019, 11, 8428.

[38] X. Lu, H. Y. Tuan, J. Chen, Z. Y. Li, B. A. Korgel, Y. Xia, J. Am. Chem. Soc. 2007, $129,1733$.

[39] K. Yang, H. Peng, Y. Wen, N. Li, Appl. Surf. Sci. 2010, 256, 3093.

[40] S. Wei, Y. Yang, X. Kang, L. Wang, L. Huang, D. Pan, Inorg. Chem. 2017, 56, 2596.

[41] S.-Q. Sun, X.-C. Hua, Q.-W. Liu, T.-T. Wang, W. Luo, Y.-J. Zhang, L.-S. Liao, M.-K. Fung, J. Mater. Chem. C 2019, 7, 2905.

[42] J. Zhang, L. Fan, J. Li, X. Liu, R. Wang, L. Wang, G. Tu, Nano Res. 2019, 12, 121. 


\section{WILEY-VCH}

[43] H. Chen, K. Ding, L. Fan, W. Liu, R. Zhang, S. Xiang, Q. Zhang, L. Wang, ACS Appl. Mater. Interfaces 2018, 10, 29076.

[44] Y.-H. Kim, G.-H. Lee, Y.-T. Kim, C. Wolf, H. J. Yun, W. Kwon, C. G. Park, T. W. Lee, Nano Energy 2017, 38, 51.

[45] M. K. Gangishetty, S. Hou, Q. Quan, D. N. Congreve, Adv. Mater. 2018, 30, 1706226.

[46] M. W. Thesen, B. Höfer, M. Debeaux, S. Janietz, A. Wedel, A. Köhler, H.-H. Johannes, H. Krueger, Journal of Polymer Science Part A: Polymer Chemistry 2010, 48, 3417.

[47] Z. Gao, C. S. Lee, I. Bello, S. T. Lee, R.-M. Chen, T.-Y. Luh, J. Shi, C. W. Tang, Appl. Phys. Lett. 1999, 74, 865.

[48] J. Jia, L. Zhu, Y. Wei, Z. Wu, H. Xu, D. Ding, R. Chen, D. Ma, W. Huang, J. Mater. Chem. C 2015, 3, 4890.

[49] A. Fakharuddin, W. Qiu, G. Croes, A. Devižis, R. Gegevičius, A. Vakhnin, C. Rolin, J. Genoe, R. Gehlhaar, A. Kadashchuk, V. Gulbinas, P. Heremans, Adv. Funct. Mater. 2019, 29, 1904101.

[50] C. G. Bischak, C. L. Hetherington, H. Wu, S. Aloni, D. F. Ogletree, D. T. Limmer, N. S. Ginsberg, Nano Lett. 2017, 17, 1028. 


\section{WILEY-VCH}
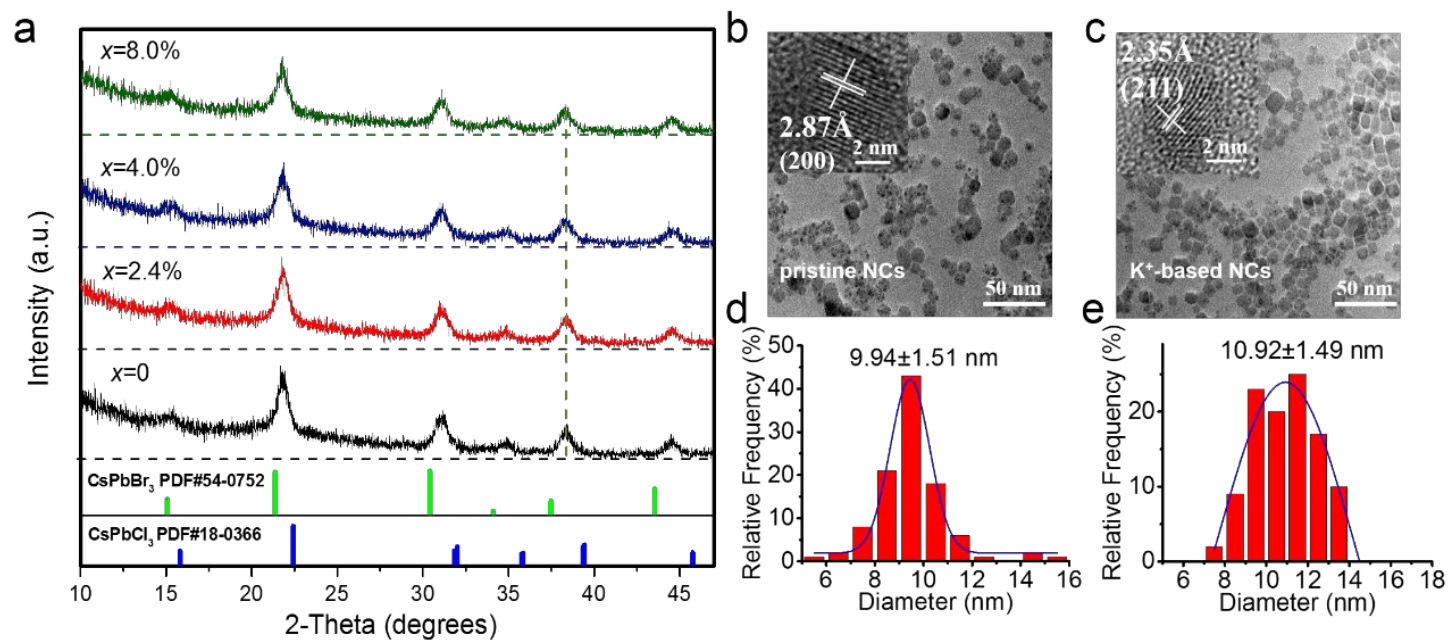

Figure 1. a) XRD patterns of perovskite films with different $x$ value and standard XRD patterns of $\mathrm{CsPbr}_{3}$ and $\mathrm{CsPbCl}_{3}$. b, c) TEM images of pristine and $\mathrm{K}^{+}$-based (4.0\% in terms of nominal $\mathrm{K}^{+}$ratio) NCs, the insets show the corresponding high-resolution TEM images. d, e) NCs size distribution diagrams of pristine and $\mathrm{K}^{+}$-based $\left(4.0 \%\right.$ in terms of nominal $\mathrm{K}^{+}$ratio) $\mathrm{NCs}$. 


\section{WILEY-VCH}
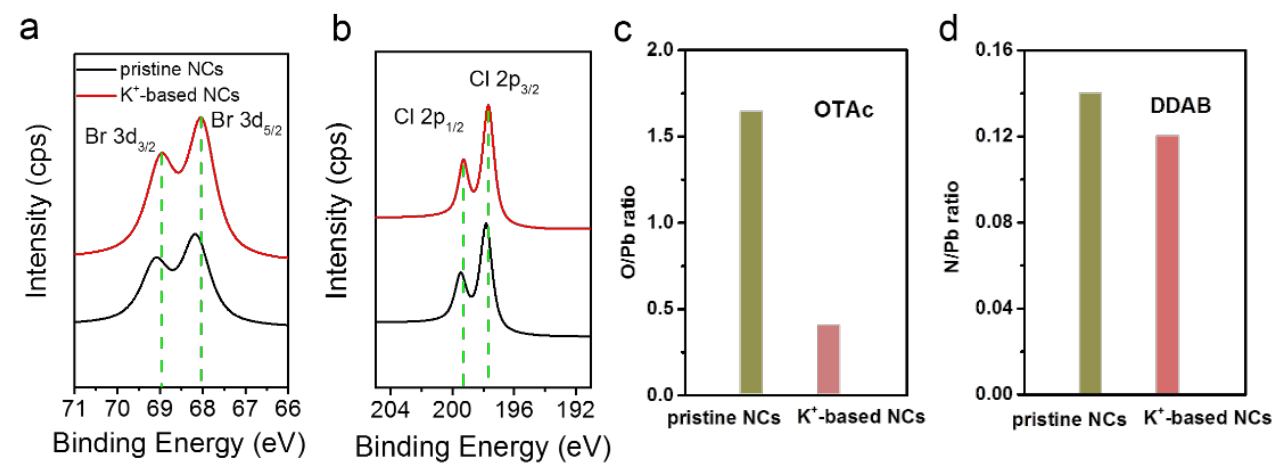

e

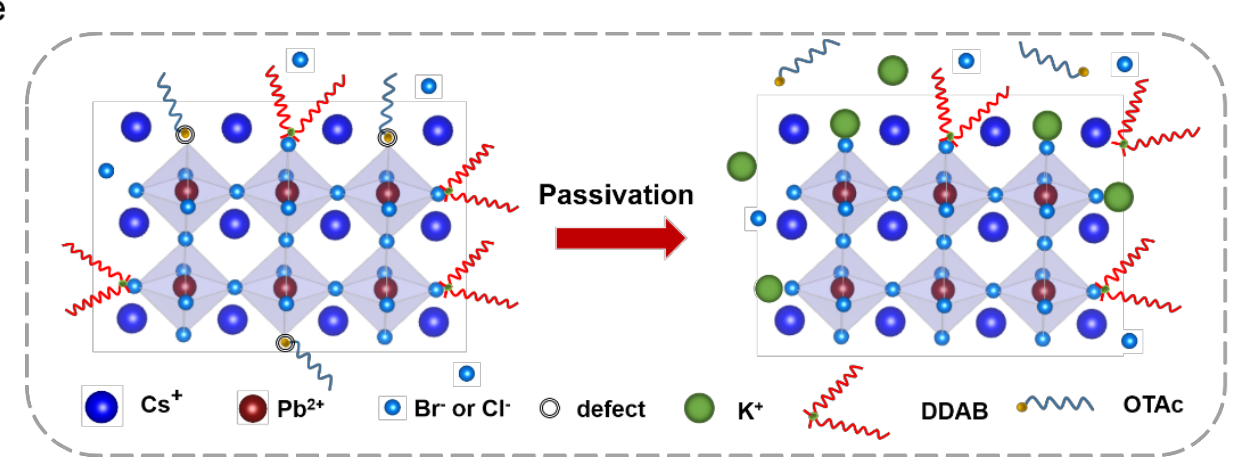

Figure 2. Zoomed in XPS spectra of pristine and $\mathrm{K}^{+}$-based (4.0\% in terms of nominal $\mathrm{K}^{+}$ratio) NCs: a) $\mathrm{Br} 3 \mathrm{~d}$, b) $\mathrm{Cl} 2 \mathrm{p}$. The atomic ratio of c) $\mathrm{O} / \mathrm{Pb}$ and d) $\mathrm{N} / \mathrm{Pb}$ for pristine and $\mathrm{K}^{+}$-based $(4.0 \%$ in terms of nominal $\mathrm{K}^{+}$ratio) NCs. e) Schematic illustration of potassium passivation. 


\section{WILEY-VCH}
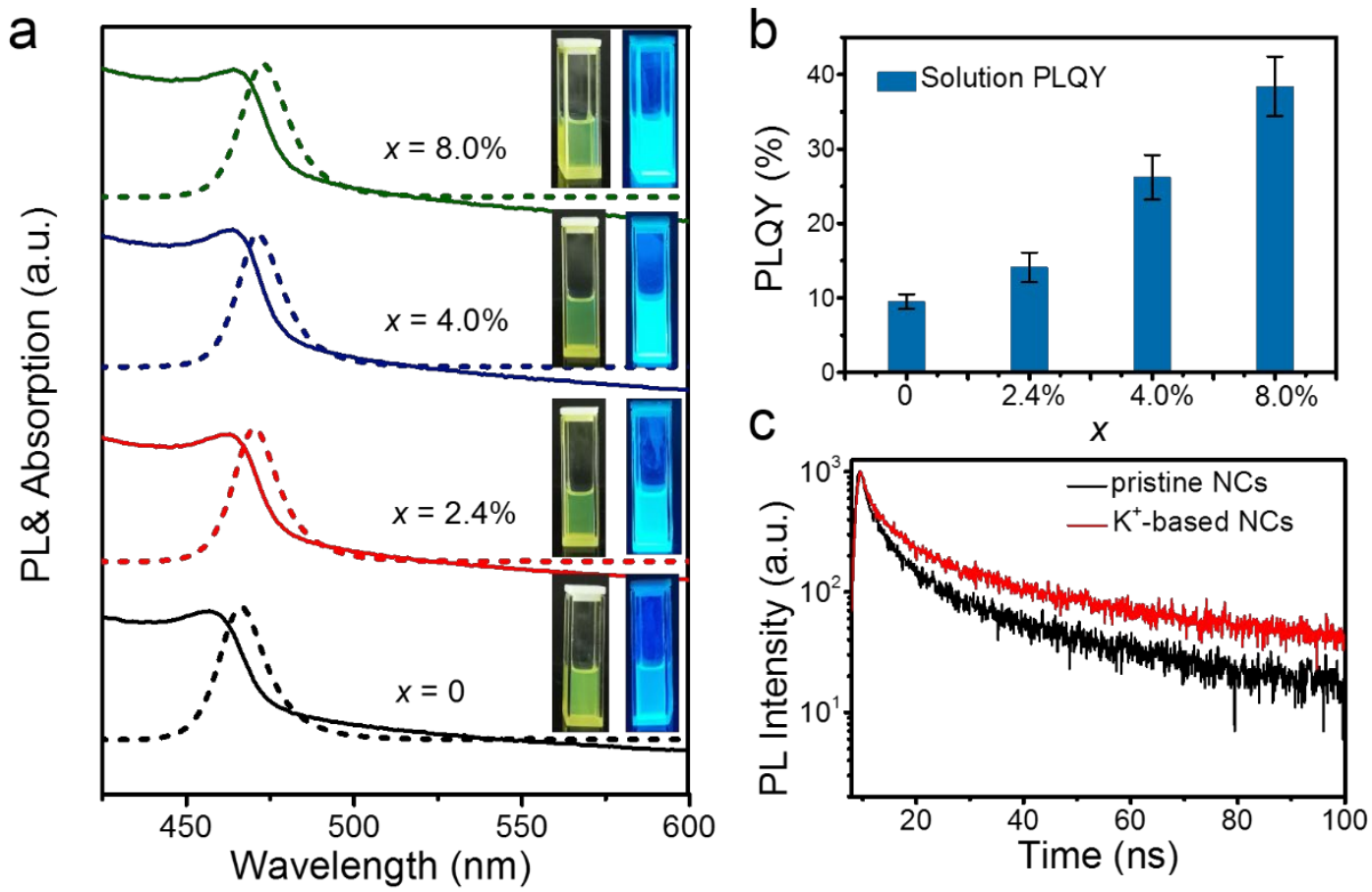

Figure 3. a) PL and UV-Vis absorption spectra of perovskite NC solutions with different $x$ value. Insets in the right display the corresponding perovskite solutions under daylight and $365 \mathrm{~nm}$ UV lamp. b) PLQY of perovskite NC solutions with different $x$ value. c) PL decay spectra of pristine and $\mathrm{K}^{+}$-based $\left(4.0 \%\right.$ in terms of nominal $\mathrm{K}^{+}$ratio) $\mathrm{NCs}$. 


\section{WILEY-VCH}

a

C
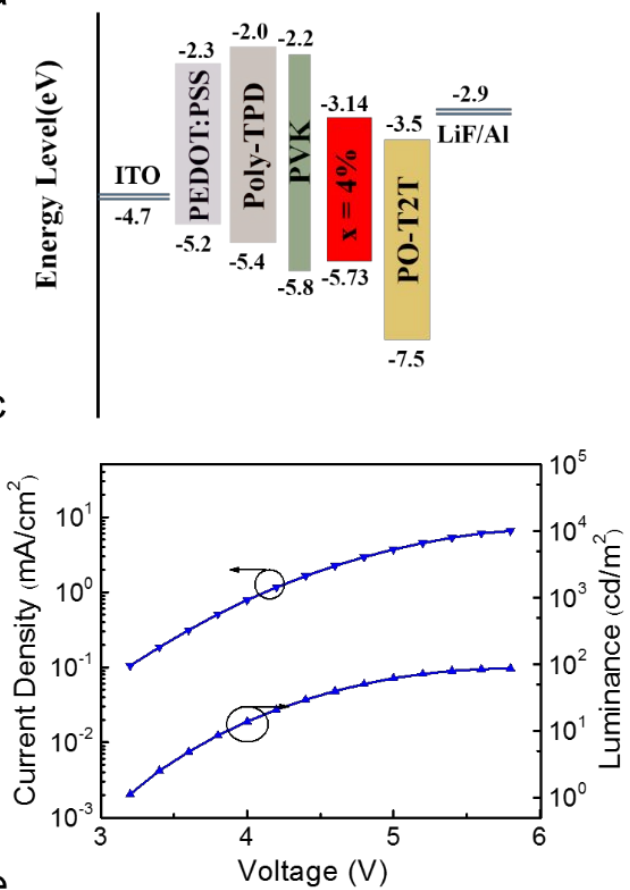

e

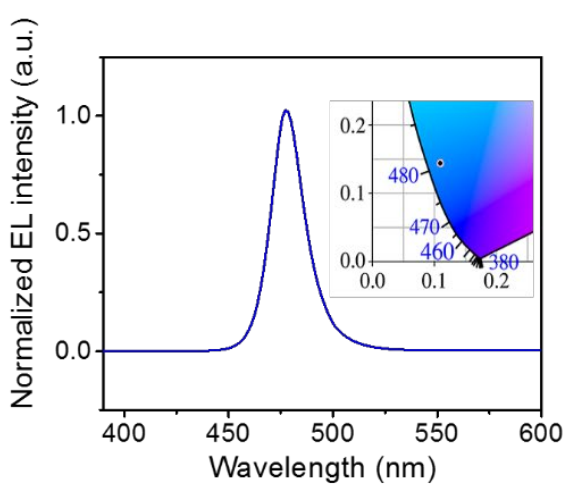

b

d
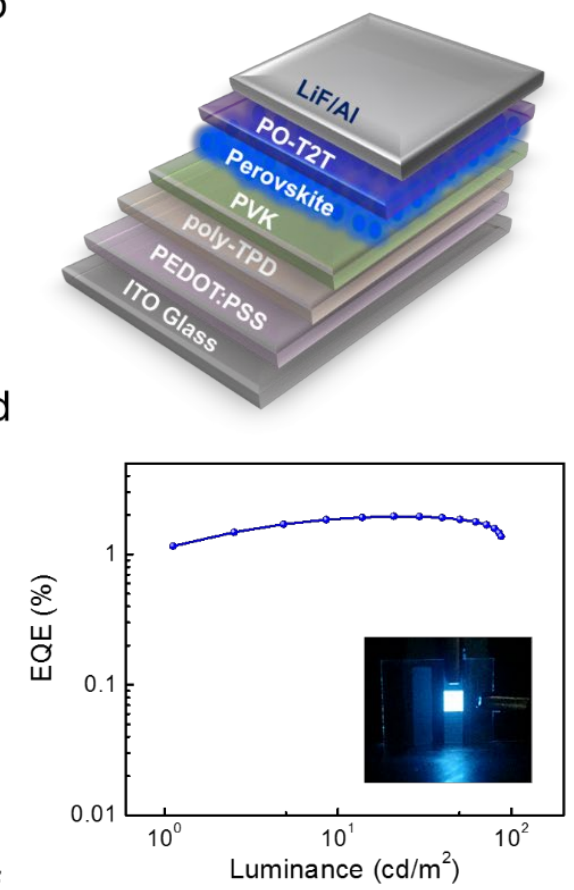

f

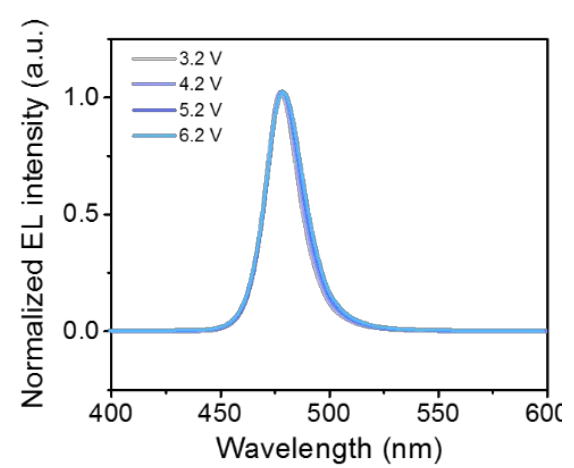

Figure 4. a) Energy level diagram and b) device structure of the LED device. c) Current densityvoltage-luminance curves and d) EQE-luminance curve for LED device based on $x=4.0 \%$. e) EL spectra of LED based on $x=4.0 \%$ driven at voltage of $3.2 \mathrm{~V}$ and inset shows the corresponding CIE coordination. f) EL spectra of the device driven at different voltage. 


\section{WILEY-VCH}

Table 1. Summarized the detail electrical output parameters of PeLEDs with different $x$ value.

\begin{tabular}{cccccc}
\hline Sample & EL peak [nm] & Max. L. [cd $\left./ \mathrm{m}^{2}\right]$ & Max. EQE [\%] & FWHM [nm] & CIE [x, y] \\
\hline $\mathrm{x}=0$ & 467 & 29.31 & 0.23 & 20 & $(0.126,0.071)$ \\
$\mathrm{x}=2.4 \%$ & 473 & 112.00 & 0.57 & 20 & $(0.113,0.102)$ \\
$\mathrm{x}=4.0 \%$ & 476 & 212.90 & 1.19 & 20 & $(0.106,0.119)$ \\
$\mathrm{x}=8.0 \%$ & 478 & 399.20 & 0.82 & 19 & $(0.099,0.140)$ \\
\hline
\end{tabular}




\section{WILEY-VCH}

Table 2. Summarized the detail electrical output parameters of PeLEDs with different ETL and HTL.

\begin{tabular}{cccccc}
\hline Device & EL peak [nm] & Max. L. [cd/m²] & Max. EQE [\%] & FWHM [nm] & CIE [x, y] \\
\hline A & 476 & 212.90 & $1.19 \%$ & 20 & $(0.106,0.119)$ \\
B & 477 & 92.56 & $1.63 \%$ & 19 & $(0.126,0.159)$ \\
C & 477 & 86.95 & $1.96 \%$ & 19 & $(0.109,0.144)$ \\
\hline
\end{tabular}




\section{WILEY-VCH}

\section{TOC}

\section{By introducing $\mathrm{K}^{+}$into $\mathrm{CsPb}(\mathrm{Br} / \mathrm{Cl})_{3}$ nanocrystals $(\mathrm{NCs})$ to reduce the non-radiative}

recombination and the amount of insulating organic ligand, we achieved high-quality blue perovskite NCs. With further engineering the device structure to improve the hole injection rate, a spectrally stable and efficient blue PeLED with a maximum external quantum efficiency of $1.96 \%$ at the emission peak of $477 \mathrm{~nm}$ is fabricated. The high-quality of $\mathrm{CsPb}(\mathrm{Br} / \mathrm{Cl})_{3} \mathrm{NCs}$ resulted from the potassium passivation strategy and the optimized device structure due to the balanced charge injection together create the outstanding blue $\mathrm{CsPb}(\mathrm{Br} / \mathrm{Cl})_{3} \mathrm{NCs}$ LEDs.

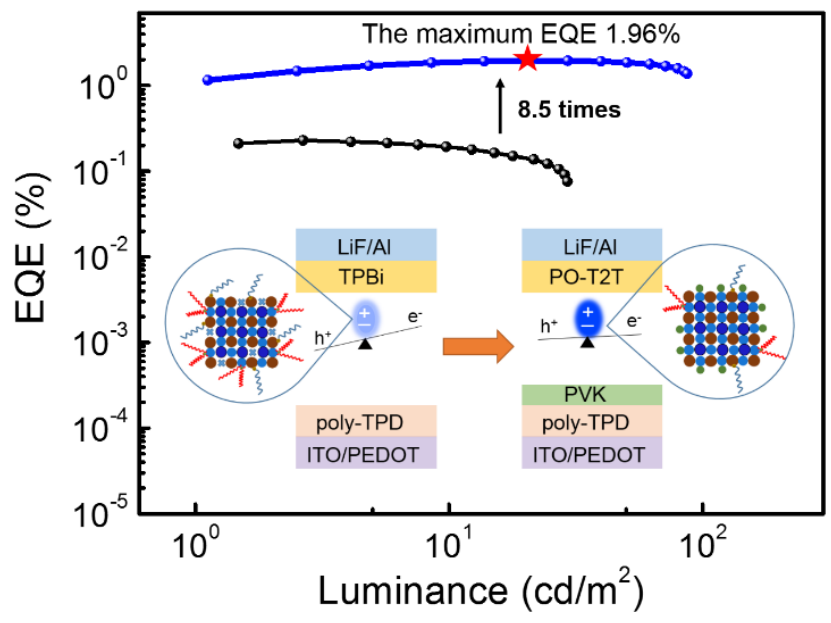

